\title{
Life cycle, growth characteristics and host cell response of Rickettsia helvetica in a Vero cell line
}

\author{
Karin Elfving $\cdot$ Agneta Lukinius $・$ Kenneth Nilsson
}

Received: 20 September 2011/Accepted: 12 November 2011/Published online: 25 November 2011

(C) The Author(s) 2011. This article is published with open access at Springerlink.com

\begin{abstract}
Rickettsia helvetica, a spotted fever rickettsia and emerging pathogen with Ixodes ricinus ticks as the main vector, is an agent of human disease and may cause febrile illness as well as meningitis. In three parallel series the isolated standard type of $R$. helvetica, obtained from a PCR-positive I. ricinus tick, was high-passaged and propagated in a Vero cell line. By using quantitative real-time PCR, the generation time from inoculation to stationary phase of growth was calculated to $20-22 \mathrm{~h}$. In the static cultivation system the stationary phase was observed from the seventh day after inoculation, and there was no observed degradation of $R$. helvetica DNA during the 14 days studied. Microscopy showed that the organisms invaded the host cells rapidly and were primarily found free in the cytoplasm and only occasionally located in the nucleus. Four days after inoculation some of the host cells were broken and many indifferent stages of cytoplasmic organic decomposition were seen. However the $R$. helvetica organism did not show any morphologic alterations and the number of organisms was stable after the replication peak which may indicate that $R$. helvetica is adapted to growth in a Vero cell line and/or that the phase of degradation occurs later than the 14 days studied. The findings differ from what has been reported for
\end{abstract}

\author{
K. Elfving · K. Nilsson $(\square)$ \\ Department of Medical Sciences, Unit of Clinical Bacteriology, \\ Uppsala University, 75185 Uppsala, Sweden \\ e-mail: kenneth.nilsson@medsci.uu.se \\ K. Elfving · K. Nilsson \\ Center of Clinical Research, Dalarna, 79182 Falun, Sweden \\ K. Elfving · K. Nilsson \\ Department of Clinical Microbiology, Falu Hospital, 79182 Falun, Sweden
}

\section{A. Lukinius}

Department of Immunology, Genetics and Pathology, Rudbeck Laboratory, Uppsala University, 75185 Uppsala, Sweden

K. Nilsson

Department of Medical Sciences, Unit of Infectious Diseases,

Uppsala University, 75185 Uppsala, Sweden 
other rickettsiae of the spotted fever group and may be of importance for invasiveness and virulence of $R$. helvetica.

Keywords Rickettsia helvetica $\cdot$ qPCR $\cdot$ Vero cells $\cdot$ Life cycle $\cdot$ Ultrastructure

\section{Introduction}

Rickettsiae are obligate intracellular bacteria that require a host cell to replicate (Raoult and Roux 1997). Eight tick-borne species or subspecies within the spotted fever group (SFG) of the genus Rickettsia have been described as emerging pathogens in Southern and Eastern Europe (Broqui et al. 2007). Furthermore, of the non-tick-borne species, Rickettsia felis, associated with cat fleas, is an emerging human pathogen (Rolain et al. 2003; Lindblom et al. 2010) and the mite-transmitted Rickettsia akari, the agent of rickettsialpox, is also known to be prevalent in Europe (Broqui et al. 2007).

Rickettsia helvetica, first isolated in Ixodes ricinus ticks in Switzerland (Burgdorfer et al. 1979) and later found in many European and Asian countries (Beati et al. 1993; Fournier et al. 2002), is the only tick-transmitted rickettsia reported from Sweden (Nilsson et al. 1999a; Severinsson et al. 2010). Ixodes ricinus is the main vector and natural reservoir, but the organism has recently also been found in Dermacentor reticulates ticks (Dobec et al. 2009). A handful of patients with a serology-based diagnosis have presented a mild, self-limited disease associated with fever, headache and myalgia, but a more severe clinical picture, sometimes with perimyocarditis as well as subacute meningitis, has been reported (Fournier et al. 2004; Nilsson et al. 1999b, 2010; Nilsson 2009).

The study was conducted in order to increase knowledge regarding growth characteristics of and host cell responses to $R$. helvetica. The standard type of $R$. helvetica was propagated in a Vero cell line in a static cultivation system in three parallel series. The mean values of the number of rickettsial DNA copies based on the gltA gene of two replicates were determined using quantitative real-time PCR (qPCR) and the kinetics of the multiplication was calculated. The third replicate was used for light and transmission electron microscopic (TEM) examinations of the morphology and ultrastructural changes in the organism and host cell. The study contributes to our understanding of the growth kinetic and host cell interactions which may be relevant for the pathogenicity and virulence of $R$. helvetica.

\section{Materials and methods}

Collection of ticks and isolation of rickettsiae

Fifty I. ricinus ticks were collected by random blanket-dragging from vegetation and tested using PCR for $17 \mathrm{kDa}$ and 16S rRNA genes (Leitner et al. 2002; Nilsson et al. 1997). A hemolymph portion of each PCR-positive tick was inoculated into Vero cells in a shell vial assay and incubated at $32^{\circ} \mathrm{C}$ and $5 \% \mathrm{CO}_{2}$. All cell lines and reagents were checked every week for bacterial contamination. One isolate of rickettsia, obtained from a PCR-positive tick, was high passaged (standard type); cells were harvested in pellets, resuspended in freezing medium, frozen in liquid notrigen and later used for the life cycle study. The growth of the rickettsiae was demonstrated using Gimenez staining (Gimenez 1964) 
and immunofluorescence using an anti-rickettsial rabbit antiserum and FITC $\gamma$-chainspecific anti-rabbit Ig (DakoCytomation Denmark A/S F0205).

Inoculation and culture procedure

With the exception of the centrifugation step, all work was carried out in a laminar flow hood. In three parallel series, $R$. helvetica was cultivated in Vero cell monolayers in shell vials of a maximum volume of $5 \mathrm{ml}$. Used as inoculums was a suspension of lysed cells from cultivation bottles already growing $R$. helvetica. Using $\mathrm{qPCR}$, the number of copies in the inoculum suspension was calculated to 5,000 copies per shell vial inoculated in $4 \mathrm{ml}$ Eagles minimal essential medium, containing $10 \%$ foetal calf serum and $1 \%$ L-glutamine, and the vials were centrifuged at $700 \mathrm{rpm}$ for $1 \mathrm{~h}$ (Allegra $\mathrm{x}-22 \mathrm{R}$, Beckman Coulter) The cell cultures were then incubated in a humid cell chamber in $5 \% \mathrm{CO}_{2}, 32^{\circ} \mathrm{C}$ for 14 days to allow rickettsiae to multiply. The growth medium was not replaced during the cultivation period and was checked continuously for bacterial contamination. The entire content of each well, including medium and cell layer, was harvested each $24 \mathrm{~h}$ interval of infection, and for each day, the rickettsia content from two wells was frozen in $-70^{\circ} \mathrm{C}$ and later quantified using qPCR. The content from the third well was stored in buffer containing $2 \%$ glutaraldehyde for later examination using TEM.

DNA extraction, PCR amplification and quantification

The harvested cells and bacteria from each well in the two parallel cultivation series were centrifuged at 17,000 rpm (Labcentrifuge, Sigma 4-15 K) for $20 \mathrm{~min}$ to capture all bacteria in both the cells and free in culture medium, the supernatant was discarded and the pellet was resuspended in $200 \mu \mathrm{l}$ PBS. DNA extraction was performed in a MagNA Pure LC according to the manufacturer's instruction (Roche). For quantification of DNA copies in the cell line, a PCR, based on the gltA gene (Stenos et al. 2005), was performed in a Light Cycler 2.0 Real-Time PCR System (Roche) using an LC Taqman Master kit (Roche). Sterile water was included as the negative control in each amplification trial.

Plasmid standard

A standard calibration curve was made from the plasmid pCR4-TOPO cloning vector containing the cloned glt A fragment (nt1126-1199) of R. helvetica (standard type). The plasmid standard was a tenfold dilution series containing $1.5-1.5 \times 10^{8}$ copies (Figs. 1, 2) of the R. helvetica gltA gene fragment. The standard curve was used for the quantification of $R$. helvetica organisms in each well from each day of infection.

\section{Immunohistochemistry}

The infected cell culture was applied by dip-pen point to microscope slides, dried, fixed in acetone, and incubated with a specific anti-rickettsial rabbit antiserum as previously described (Nilsson et al. 1999b). Immunoglobulin G (IgG) antibodies were detected by fluorescein isothiocyanate-conjugated (FITC) $\gamma$-chain-specific goat anti-human globulin (Dako, Glostrup, Denmark). Appropriate positive and negative controls were added to each set of slides. A human serum with an end-point titer of 1/640 for $R$. helvetica was used as positive control. 


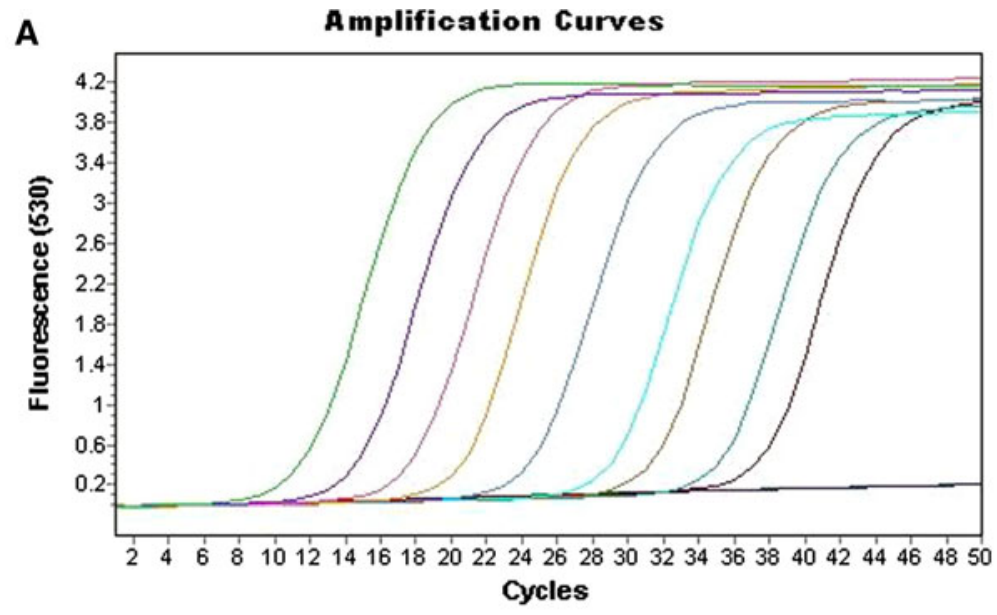

B Standard Curve

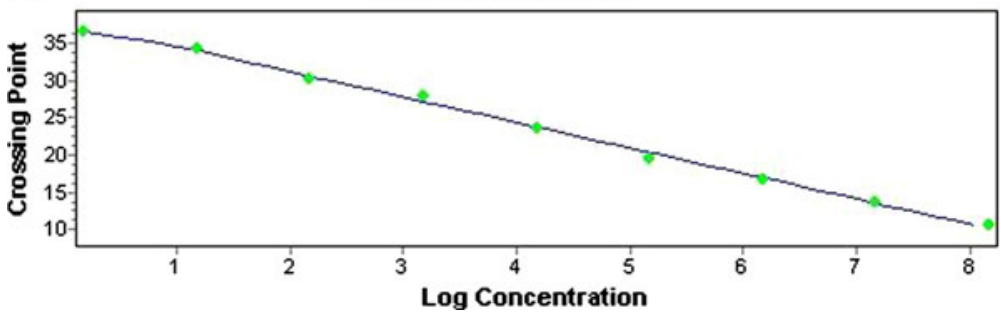

Fig. 1 a qPCR amplification in a dilution series of the plasmid pCR4-TOPO cloning vector containing the inserted gltA fragment of Rickettsia helvetica (standard type). b Standard calibration curve for R. helvetica plasmid pCR4-TOPO cloning vector showing linearity in the dilution series between 1.5 and $1.5 \times 10^{8}$ copies (Error 0.0142, Efficiency 1.961, $\mathrm{R}^{2} 0.997, \mathrm{y}=-3.34 \mathrm{x}+37.68$ )

\section{Mean value of number of Rickettsia copies from two parallel cultivation series}

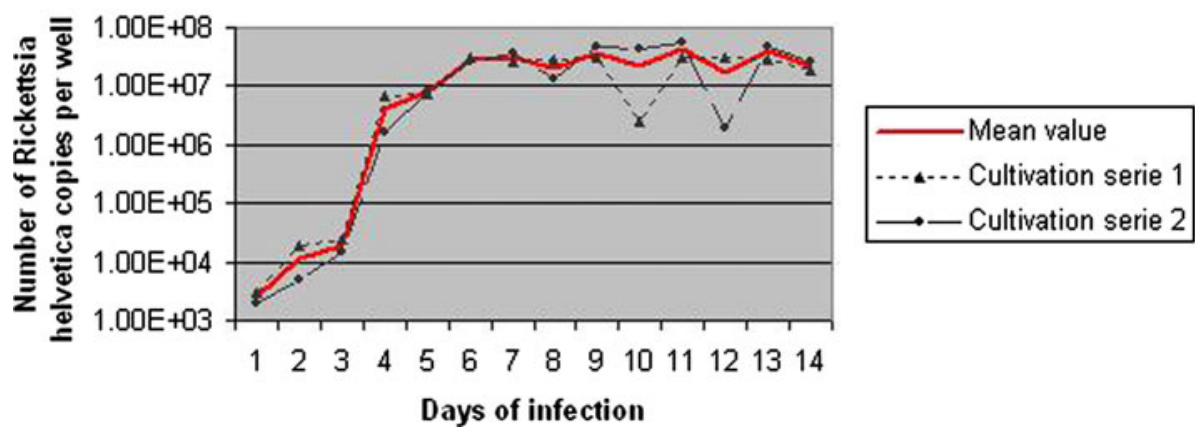

Fig. 2 qPCR of content from each well with actual and mean values of the number of Rickettsia helvetica copies in two parallel cultivation series in Vero cells for 14 days 
Transmission electron microscopy (TEM)

For morphological analysis, the content from wells representing day 1, 3, 5, 7, 9, 11, and 13 were processed by fixation in $2 \%$ glutaraldehyde in a $0.1 \mathrm{M}$ sodium cacodylate buffer ( $\mathrm{pH}$ 7.2), supplemented with $0.1 \mathrm{M}$ sucrose, postfixation in $1 \%$ osmium tetroxide, dehydration in ethanol and embedding in epoxy resin Agar 100 (Agar Scientific, Stansted, UK). Ultrathin sections were placed on formvar-coated copper grids, contrasted with $4 \%$ uranyl acetate and Reynolds lead citrate, and analysed in a Tecnai Bio TWIN electron microscope (FEI, Eindhoven, Netherlands). For immunocytochemical labelling, the Vero cells were processed according to a low-temperature protocol, i.e. short fixation in $4 \%$ paraformaldehyde/ $0.5 \%$ glutaraldehyde in PBS at $4{ }^{\circ} \mathrm{C}$, rapid dehydration in $50-95 \%$ ethanol and lowering of the temperature to $-20^{\circ} \mathrm{C}$, infiltration and polymerization (UV-light) in Lowicryl $\mathrm{K} 4 \mathrm{M}$ at $-20^{\circ} \mathrm{C}$ (Lukinius et al. 1989). Fifty nm sections were placed on Formvar-coated gold grids. All sections were blocked for unspecific binding by applying $5 \%$ bovine serum albumin (BSA, Sigma, St. Louis, MO) for $30 \mathrm{~min}$, followed by incubation overnight at $4^{\circ} \mathrm{C}$ with a specific antirickettsial rabbit antiserum diluted 1:50 in $0.05 \mathrm{M}$ TBS $\mathrm{pH} 7.2$, supplemented with $0.1 \%$ BSA. After rinsing in $0.05 \mathrm{M}$ TBS, pH 7.2, with $0.2 \%$ BSA, and in TBS, pH 8.2, with $1 \% \mathrm{BSA}$, the sections were incubated with 10 and $15 \mathrm{~nm}$ gold-conjugated goat antirabbit IgG (GAR-G10 or GAR-G15; Amersham International, Amersham, Bucks, UK) diluted 1:20 in TBS, $\mathrm{pH} 8.2$, with 1\% BSA, for $2 \mathrm{~h}$. The sections were then rinsed and contrasted with $4 \%$ uranyl acetate and Reynolds lead citrate. Immunogold labeling of some of the morphologically processed samples was also performed. These sections were initially etched in $1 \mathrm{~N} \mathrm{NaOH}$ for $1 \mathrm{~min}$ to compensate for the epoxy resin embedding (Lukinius et al. 1989). Negative controls were obtained by excluding the primary antiserum or replacing it with nonimmune serum.

\section{Results}

Tick isolation

Of the 50 tested I. ricinus ticks, three were positive by PCR for $17 \mathrm{kDa}$ and $16 \mathrm{~S}$ rRNA genes, resulting in an infection rate of $6 \%$. The sequences obtained from these ticks were identical to those of $R$. helvetica (GenBank accession no EU407139, EU407140). One isolate was obtained from a PCR-positive tick. The growth of the rickettsiae was demonstrated by immunofluorescence and Gimenez staining, and PCR followed by sequencing and Blast analysis showed that the fragments were identical to those of $R$. helvetica.

\section{Plasmid standard}

Figure 1 presents the quantification curve of the serially diluted pCR4-TOPO plasmid containing the cloned 74-bp fragment of $R$. helvetica glt $\mathrm{A}$ gene. The minimum detection limit for the plasmid was 1-2 copies when using the qPCR assay (Stenos et al. 2005). Reagent controls containing no DNA were not amplified. The plasmid standard showed linearity within the whole range of the dilution series (Fig. 1a-b). 
Cultivation and quantification of rickettsia

The number of $R$. helvetica copies per well was calculated using the qPCR assay and compared with the standard calibration curve. The parallel cultivation series were analysed separately, and the actual values and mean values of rickettsial copies are shown in Fig. 2. $R$. helvetica grew rapidly in Vero cell culture, and the curve demonstrates an initial shortlag phase (day 1-3) followed by an exponential increase in rickettsial copies. The highest replication rate of bacteria was recorded on the fourth day post-infection before a stationary phase established on the sixth (series one) to seventh (series two) day of infection. The generation times, including both series, were calculated to be $15.6 \mathrm{~h}$ during the first 6 days and $26.4 \mathrm{~h}$ until day seven. The growth curve for series 2 levels out between six and seven days, which is why the generation time on average for both series falls between 20 and $22 \mathrm{~h}$. On the eleventh day, the highest point of multiplication was recorded. At that time, the copy number of rickettsiae was $4.3 \times 10^{7}$ bacteria per well, which is 8,600 times greater than the inoculum level of 5,000 copies.

Transmission electron and light microscopy

The ultrastructural findings confirmed that $R$. helvetica in a Vero cell line on the first and second day post-infection invaded the cytoplasm through phagocytosis and later escaped the phagocytic vacuoles through lysis. Binary fission was observed from the third day postinfection. $R$. helvetica invaded the host cell, and light and electron microscopy demonstrated its presence in large numbers in the cell cytoplasm (Figs. 3a, 4a). Sparse penetration of the nuclear membrane was occasionally observed (Fig. 3a-b). Filopodia was observed in some of the cells (Fig. 3c). Intracellular rickettsiae grew either in small clusters or as solitary organisms and were either spherical or elongated in shape. A cell wall characteristic for rickettsiae, consisting of inner and outer leaflets of glycolipid membranes, enveloped each organism (Fig. 4a). At 4 days postinfection decomposition of Vero cell cytoplasmic organells were seen in many of the host cells and debris from collapsed cells and free $R$. helvetica were revealed (Fig. $4 \mathrm{a}-\mathrm{b}$ ).

Immunocytochemical gold-labelling revealed the presence of $R$. helvetica and demonstrated a specific labelling primarily in the outermost leaflets and less in the matrix (Fig. 4b). The positive labelling was positive and negative controls were negative.
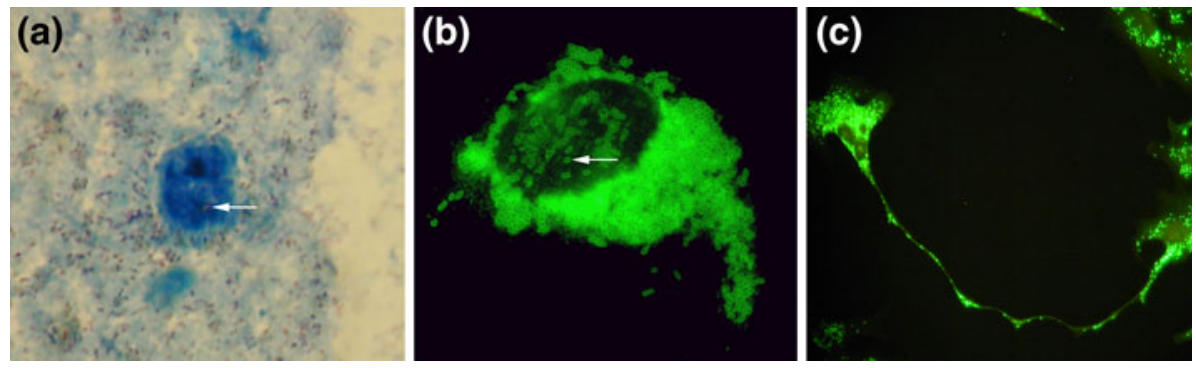

Fig. 3 Fourth day of Rickettsia helvetica infected Vero cells. a Vero cell with Gimenez stained rickettsiae in the cytoplasm. Single bacteria can be seen in the nucleus (arrow) $\times 400$. b Fluorescent photomicrograph of infected Vero cell where a sparse number of bacteria are seen in the nucleus $\times 1000$. c Fluorescent photomicrograph of a filopodia filled with fluorescent coccobacillary rickettsiae stained with specific rabbit anti-rickettsia antibodies $\times 200$ 

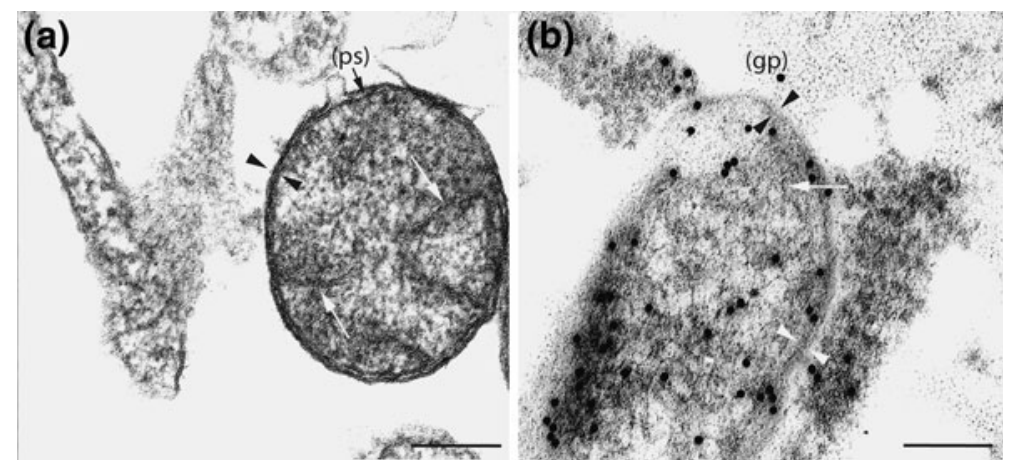

Fig. 4 Fourth to fifth day of Rickettsia helvetica infected Vero cells. a Morphology of R. helvetica in partly decomposed host cells. Note the leaflets (arrow heads) and inner plasma membrane enclosing the periplasmatic space (ps). Fibrillate nucleic acid is clearly visible (long arrows) $\times 120,000$. Bar $150 \mathrm{~nm}$. b Anti-rickettsia antibodies with gold particles $(\mathrm{gp})(15 \mathrm{~nm})$ on Lowicryl-embedded cells. Leaflets and plasma membrane are visible (arrows heads) as well as fibrillar nucleic acid (long arrows). The immunoreaction is mainly located along the membrane/leaflet part of the rickettsia but sparsely scattered all over the organism $\times 120,000$. Bar $150 \mathrm{~nm}$

\section{Discussion}

Bacterial growth is generally characterized by four phases: lag, exponential, stationary and death. In our experimental conditions, after inoculation of the standard type of $R$. helvetica, almost all bacteria were taken up into the host cells. There was a lag phase of 2-3 days, after which followed exponential growth of the organism with the maximum number of replicates on the sixth to seventh day, and then a stationary phase was seen (Fig. 2). Similar results for Rickettsia slovaca have shown a maximum of multiplication on the second day post-infection for the wild type and on the fourth day for the standard type (Boldis et al. 2009). In that case, R. slovaca was propagated in both Vero and L929 cells with similar results $(20.3 \mathrm{~h} /$ Vero cells and $21.87 \mathrm{~h} / \mathrm{L} 929$ cells $)$, but $R$. conorii have been shown to have higher propagation rates when using L929 cells (Balraj et al. 2009). For Rickettsia rickettsii (wild type) a maximum on the second to third day post-infection was observed (Eremeeva et al. 2003). Concerning the lag phase for R. helvetica in our study, the number of bacteria in the inoculum was considerably lower than used in the experiments for $R$. slovaca and $R$. rickettsii, which makes it likely that the lag phase for $R$. helvetica will be longer and more pronounced than for the others.

For $R$. helvetica, the exponential phase between day three and seven was initially vigorous, after which a stationary growth phase was noted and no death phase was demonstrated (Fig. 2). The generation time for R. helvetica $(20-22 \mathrm{~h})$ during the lag and exponential phase was similar to the reported time for $R$. slovaca $(20.3 \mathrm{~h} /$ Vero cells and $21.87 \mathrm{~h} / \mathrm{L} 292$ cells) (Boldis and Spitalska 2010). However, the total number of copies was considerably higher for $R$. helvetica at the time of transition to stationary phase.

Concerning $R$. rickettsii (wild type) and $R$. slovaca (standard type), the death phase started on the third to fifth day, the same or 1 day after the replication peak, with gradual degradation of DNA (Boldis et al. 2009; Eremeeva et al. 2003). During the 14 days of culturing and 7 days after the peak of replication, there was no observed degradation of DNA for $R$. helvetica. The findings may indicate that our strain of $R$. helvetica may be more stable than what has been reported for $R$. slovaca and $R$. rickettsii and/or is adapted to 
growth in a Vero cell line. Another reason, in our study, could be that the centrifugation step of the contents of each well captured all bacteria in both cells and in culture medium explaining that few losses in DNA copies were seen. The significance of this observation needs to be further studied to understand its impact on bacterial invasiveness and virulence. In electron microscopy, no bacteria with vacuoles typical for the bacteria in the stationary phase were seen, contrary to what was demonstrated in rickettsiae in ticks (Nilsson et al. 1997) which also indicates that the phase of degradation occurs later than the 14 days studied. Besides the effect on growth, the host cell is of importance to both rickettsial adherence and host cell penetration (Uchiyama 2005).

One of the features and taxonomic criterion of SFG rickettsiae is their ability to grow intranuclearly. $R$. helvetica has previously been identified intranucleary in rectal ampule tissues of infected I. ricinus (Burgdorfer et al. 1979). In the present study, nuclear localization was confirmed by microscopy and immunofluorecens (Fig. 3a-b), but the incidence of this phenomenon was low and probably not optimal as a criterion for differentiation between rickettsial species.

Rickettsia replicates through binary fission and invades the nearby cells by inducing filopodia formation. Via filopodia propulsion, the rickettsiae are either released extracellularly or spread into the adjacent cell (Walker and Ismail 2008). The phenomenon was demonstrated in some of the cells with filopodia measuring a length equivalent to seven Vero cell diameters (Fig. 3c). Binary fission was frequently observed through TEM. Most of the rickettsiae were free in the cytoplasm and were by TEM or immunogold-labelling easily distinguished from host cell organelles as mitochondria and various granules, which never multiply by binary fission or have electron-dense outer membranes. Moreover, the immunogold-labelled anti-rickettsial antibody showed a specific immunoreaction with labelling throughout the rickettsial matrix, but mainly located along the membrane/leaflet part of the organism (Fig. 4b).

The role of humans in the life cycle of SFG rickettsiae is secondary, and it has for some years not been definitely proven that $R$. helvetica is pathogenic for humans but indicated by cell disintegration in cell culture and electron microscopic studies of infected human tissue (Nilsson et al. 2002). The findings in this study of decomposition of host cells and intranuclear growth underlines the pathogenic ability of $R$. helvetica. Besides, the bacterium has now been isolated from the cerebrospinal fluid in humans, confirming its invasiveness and pathogenicity and is also reported to cause aneruptive fever (Nilsson et al. 2010; Fournier et al. 2004). The symptoms of the disease may generally be somewhat milder than what has been described for Mediterranean spotted fever (Rickettsia conorii) and Rocky Mountain spotted fever ( $R$. rickettsii). This is, in this study, not reflected in lower invasiveness for $R$. helvetica in the host cells than what has been reported for other rickettsiae of the spotted fever group. Further studies of the cellular response are therefore needed to fully understand the mechanisms of $R$. helvetica's pathogenesis and virulence.

Acknowledgments The study was financially supported by grants from the Center of Clinical Research Dalarna (9028); Olle Engkvist Byggmästare Foundation (11877).

Conflict of interest The authors declare no conflict of interest.

Open Access This article is distributed under the terms of the Creative Commons Attribution Noncommercial License which permits any noncommercial use, distribution, and reproduction in any medium, provided the original author(s) and source are credited. 


\section{References}

Balraj P, Vestris D, Raoult D, Renesto P (2009) Comparison of Rickettsia conorii growth within different cell lines by real-time quantitative PCR. Clin Microbiol Infect 15:294-295

Beati L, Peter O, Burgdorfer W, Aeschlimann A, Raoult A (1993) Confirmation that Rickettsia helvetica sp. nov. is a distinct species of the spotted fever group of rickettsiae. Int J Syst Bacteriol 43:521-526

Boldis V, Spitalska E (2010) Dermacentor marginatus and Ixodes ricinus ticks versus L929 and Vero cell lines in Rickettsia slovaca life cycle evaluated by quantitative real time PCR. Exp Appl Acarol 50:353-359

Boldis V, Strus J, Kocianova E, Tusek-Znidaric M, Stefanidesova K, Schwarzova K, Kudelova M, Sekeyova Z, Spitalska E (2009) Life cycle of Rickettsia slovaca in L929 cell line studied by quantitative realtime PCR and transmission electron microscopy. FEMS Microbiol Lett 293:102-106

Broqui P, Parola P, Fournier PE, Raoult D (2007) Spotted fever rickettsioses in southern and eastern Europe. FEMS Immunol Med Microbiol 49:2-12

Burgdorfer W, Aeschlimann A, Peter O, Hayes SF, Philip RN (1979) Ixodes ricinus: vector of a hitherto undescribed spotted fever group agent in Switzerland. Acta Trop 36:357-367

Dobec M, Golubic D, Punda-Polic V, Kaeppeli F, Sievers F (2009) Rickettsia helvetica in Dermacentor reticulates ticks. Emerg Infect Dis 15:98-100

Eremeeva EE, Dash AD, Silverman DJ (2003) Evaluation of a PCR assay for quantification of Rickettsia rickettsii and closely related spotted fever group rickettsiae. J Clin Microbiol 41:5466-5472

Fournier PE, Fujita H, Takada N, Raoult N (2002) Genetic identification of rickettsiae isolated from ticks in Japan. J Clin Microbiol 40:2176-2181

Fournier PE, Allombert C, Supputamongkol Y, Caruso G, Brouqui P, Raoult D (2004) Aneruptive fever associated with antibodies to Rickettsia helvetica in Europe and Thailand. J Clin Microbiol 42:816-818

Gimenez DF (1964) Staining rickettsiae in yolk-sac cultures. Stain Technol 39:135-140

Leitner M, Yitzhaki S, Rzotkiewicz S, Keysary A (2002) Polymerase chain reaction-based diagnosis of mediterranean spotted fever in serum and tissue samples. Am J Trop Med 67:166-169

Lindblom A, Severinsson K, Nilsson K (2010) Report of two cases with subacute meningitis and review of the literature. Scand J Infect Dis 42:906-909

Lukinius A, Wilander E, Westermark GT, Engström U, Westermark P (1989) Co-localisation of islet amyloid polypeptide and insulin in the B-cell secretory granules of the human pancreatic islets. Diabetologia 32:240-244

Nilsson K (2009) Septicaemia with Rickettsia helvetica in a patient with acute febrile illness, rash and myasthenia. J Infect 58:79-82

Nilsson K, Jaenson TG, Uhnoo I, Lindquist O, Pettersson B, Uhlén M, Friman G, Påhlson C (1997) Characterization of a spotted fever group rickettsia from Ixodes ricinus ticks in Sweden. J Clin Microbiol 35:243-247

Nilsson K, Lindquist O, Liu AJ, Jaenson TG, Friman G, Påhlson C (1999a) Rickettsia helvetica in Ixodes ricinus ticks in Sweden. J Clin Microbiol 37:400-403

Nilsson K, Lindquist O, Påhlson C (1999b) Association of Rickettsia helvetica with chronic perimyocarditis in sudden cardiac death. Lancet 354:1169-1173

Nilsson K, Påhlson C, Lukinius A, Eriksson L, Nilsson L, Lindquist O (2002) Presence of Rickettsia helvetica in granulomatous tissue in patients with sarcoidosis. J Infect Dis 185:1128-1138

Nilsson K, Elfving K, Påhlson C (2010) Rickettsia helvetica in patient with meningitis, Sweden, 2006. Emerg Infect Dis 16:490-492

Raoult D, Roux V (1997) Rickettsioses as paradigms of new or emerging infectious diseases. Clin Microbiol Rev 10:694-719

Rolain JM, Franc M, Davoust B, Raoult D (2003) Molecular detection of Bartonella quintana, B. koehlerae, B. henselae, B. clarridgeiae, Rickettsia felis, and Wohlbachia pipientis in cat fleas, France. Emerg Infect Dis 9:338-342

Severinsson K, Jaenson TG, Pettersson J, Falk K, Nilsson K (2010) Detection and prevalence of Anaplasma phagocytophilum and Rickettsia helvetica in Ixodes ricinus ticks in seven study areas in Sweden. Parasit Vectors 3:66:1-7

Stenos J, Graves SR, Unsworth NB (2005) A highly sensitive and specific real-time PCR assay for the detection of spotted fever and typhus group rickettsiae. Am J Trop Med Hyg 73:1083-1085

Uchiyama T (2005) Growth of typhus group and spotted fever group rickettsiae in insect cells. Ann N Y Acad Sci 1063:215-221

Walker DH, Ismail N (2008) Emerging and re-emerging rickettsioses: endothelial cell infection and early disease events. Nat Rev Microbiol 6:375-386 (Review) 